\title{
Proof of the Double Bubble Curvature Conjecture
}

\author{
By Marilyn Daily
}

\begin{abstract}
An area minimizing double bubble in $\mathbb{R}^{n}$ is given by two (not necessarily connected) regions which have two prescribed $n$-dimensional volumes whose combined boundary has least (n-1)-dimensional area. The double bubble theorem states that such an area minimizer is necessarily given by a standard double bubble, composed of three spherical caps. This has now been proven for $n=2,3,4$, but is, for general volumes, unknown for $n \geq 5$. Here, for arbitrary $n$, we prove a conjectured lower bound on the mean curvature of a standard double bubble. This provides an alternative line of reasoning for part of the proof of the double bubble theorem in $\mathbb{R}^{3}$, as well as some new component bounds in $\mathbb{R}^{n}$.
\end{abstract}

\section{Introduction}

In $\mathbb{R}^{3}$, a standard double bubble describes the familiar surface which is formed when two spherical soap bubbles join to enclose two volumes. As Plateau empirically observed [9], such a surface consists of three spherical caps which meet at $120^{\circ}$ angles. Analogously, a standard double bubble in $\mathbb{R}^{n}$ is defined as a collection of three $(n-1)$-dimensional spherical caps which intersect at $120^{\circ}$ angles along a common $(n-2)$-dimensional sphere.

The double bubble conjecture states that in $\mathbb{R}^{n}$, a standard double bubble is the unique surface of minimal area which encloses two given volumes. This conjecture has been proven in $\mathbb{R}^{2}$ [3], $\mathbb{R}^{3}[7]$, and $\mathbb{R}^{4}[10]$, but remains an open problem in higher dimensions. A major difficulty is the possibility that one of the enclosed regions of an area minimizing double bubble may not be connected. Although a least-area surface which encloses and separates two regions of given volumes must exist [1, Theorem VI.2], the existence proof allows each enclosed region to have more than one component.

The Hutchings Basic Estimate and its corollaries provide a way to bound the number of components in each region of an area-minimizing double bubble. In particular, in a double bubble which encloses regions of volumes $v$ and $1-v$, they give us a function $K(v)$ which provides an upper bound on the number of components in the region of volume $v$.

Although computer-generated graphs of $K(v)$ have long suggested that $K(v)$ is decreasing, until now there has been no rigorous mathematical proof of this. The Curvature Conjecture

Math Subject Classifications. 53A10.

Key Words and Phrases. Double bubble conjecture, mean curvature. 
([10, Conjecture 4.10], [8, Conjecture 14.14]) provides a way to finally prove that $K(v)$ is decreasing, which will allow us to determine component bounds accordingly.

Theorem 1.1 (Curvature Conjecture). In $\mathbb{R}^{n}$, let $H_{0}, H_{1}, H_{2}$, respectively, denote the mean curvature of a sphere of volume $w$, a sphere of volume $w+1$, and the exterior of the second region of a standard double bubble of volumes $1, w$. Then

$$
2 H_{2}>H_{0}+H_{1} \text {. }
$$

The curvature conjecture was proven in $\mathbb{R}^{2}$ and in all dimensions for the case $w \geq 1$ by David Futer [4].

\section{The curvature conjecture in the double bubble problem}

First, we will place the curvature conjecture into context, by citing some results which have been proven elsewhere. A much fuller account of this history can be found in Chapter 14 of [8]. Let $A(v, w)$ denote the minimal area required to enclose and separate volumes $v, w$ in $\mathbb{R}^{n}$, let $A(v)=A(v, 0)$ denote the surface area of a sphere of volume $v$, and let $\tilde{A}(v, w)$ denote the surface area of the standard double bubble enclosing regions of volumes $v$ and $w$.

Theorem 2.1 (Hutchings Basic Estimate [6, Theorem 4.2]). Consider a minimizing double bubble of volumes $v, w$ in $\mathbb{R}^{n}$. If the first region has a component of volume $x>0$, then $A(v)[v / x]^{1 / n} \leq 2 A(v, w)-A(v+w)-A(w)$.

Corollary 2.2. In a minimizing double bubble of volumes $v$, $w$, if the region with volume $v$ consists of $k$ components, then $A(v) k^{1 / n} \leq 2 \tilde{A}(v, w)-A(v+w)-A(w)$.

Although the Curvature Conjecture has remained unproven until now, it has had the following two corollaries for a while. Corollary 2.3 asserts that when $v=1$, the preceding bound is an increasing function of $w$.

Corollary $2.3\left(\left[5\right.\right.$, Conjecture 4.9]). $\quad f(w):=\frac{2 \tilde{A}(1, w)-A(1+w)-A(w)}{A(1)}$ is increasing.

Similarly, by considering the Hutchings Basic Estimate when $w=1-v$, we get an upper bound $K(v)$ on the number of components in the first region of a $(v, 1-v)$ double bubble.

$$
K(v):=\frac{2 \tilde{A}(v, 1-v)-A(1)-A(1-v)}{A(v)} \geq k^{1 / n} .
$$

Corollary 2.4 , which is implicitly present in [8, pp. 150-151], states that this function decreases as $v$ increases.

Corollary 2.4. $K(v)=\frac{2 \tilde{A}(v, 1-v)-A(1)-A(1-v)}{A(v)}$ is decreasing.

Since $k^{1 / n} \leq K(v)$ and $K(v)$ is decreasing, an absolute upper bound for the number of components in the region with volume $v$ is given by

$$
\lim _{v \rightarrow 0}\left(\frac{2 \tilde{A}(v, 1-v)-A(1)-A(1-v)}{A(v)}\right)^{n} .
$$

Unfortunately, the upper bound which results from this limit is too large to be usable for most $n$. In $\mathbb{R}^{3}$, however, we can calculate the following useful result. 
Theorem 2.5. The smaller region of a double bubble in $\mathbb{R}^{3}$ has at most two components.

Proof. $\quad \lim _{v \rightarrow 0} K(v)=\left(\frac{5}{2}\right)^{1 / 3}$, which implies that $K(v)^{3} \leq 2.5$.

Theorem 2.6. In $\mathbb{R}^{3}$ and $\mathbb{R}^{4}$, the larger region of a double bubble is connected. In $\mathbb{R}^{n}$, the larger region of a double bubble can have at most three components.

Proof. Since $K$ is decreasing, $K\left(\frac{1}{2}\right)^{n}$ provides an upper bound for the number of components in the larger region. Since $A\left(\frac{1}{2}\right)=n \sqrt[n]{\alpha_{n}\left(\frac{1}{2}\right)^{n-1}}$,

$$
K\left(\frac{1}{2}\right)=\frac{2 n \sqrt[n]{2 \alpha_{n-1} \int_{0}^{2 \pi / 3} \sin ^{n} \theta d \theta}-A(1)-A\left(\frac{1}{2}\right)}{A\left(\frac{1}{2}\right)}=4 \sqrt[n]{\frac{\int_{0}^{2 \pi / 3} \sin ^{n} \theta d \theta}{\int_{0}^{\pi} \sin ^{n} \theta d \theta}}-\frac{2}{\sqrt[n]{2}}-1 .
$$

When $n=3$ or $n=4$, one can verify that $g\left(\frac{1}{2}\right)^{n}<2$, and it is also not difficult to show that $K\left(\frac{1}{2}\right)^{n}<4$ for all $n \in \mathbb{N}$.

Remark. In $\mathbb{R}^{3}$ and $\mathbb{R}^{4}$, one can also use Schoenfeld balancing [2] in order to show that the larger region is connected.

When $n$ is large, we must note that there is a much simpler way to bound the number of components in each region of a double bubble in $\mathbb{R}^{n}$. If we define

$$
G(v):=\frac{2[A(v)+A(1-v)]-A(1)-A(1-v)}{A(v)}=2+\left(\frac{1-v}{v}\right)^{(n-1) / n}-\left(\frac{1}{v}\right)^{(n-1) / n},
$$

then $G$ is decreasing, and the Hutchings Basic Estimate implies that $k<(G(v))^{n}$. According to the Balancing Theorem [6], if the larger region in a double bubble consists of more than $\frac{2}{3}$ of the total volume, then the larger region is connected. The following theorem then reveals that if the larger region of a double bubble is not connected, then the smaller region can have at most six components.

Theorem 2.7. In a double bubble consisting of regions of volumes $v$ and $1-v$, where the larger region is of volume $v \leq \frac{2}{3}$, the smaller region consists of at most six components.

Proof. Since $G$ is decreasing and the larger region has volume $v \leq \frac{2}{3}$, the smaller region has at most $G\left(\frac{1}{3}\right)^{n}$ components. Since $G\left(\frac{1}{3}\right)=2+2^{(n-1) / n}-3^{(n-1) / n}$, we need to prove that $\left(2+2^{(n-1) / n}-3^{(n-1) / n}\right)^{n}<7$. Equivalently, we will show that $7^{1 / n}+3 \cdot 3^{-1 / n}-2 \cdot 2^{-1 / n}>2$.

$$
\frac{d}{d x}\left(7^{1 / x}+3 \cdot 3^{-1 / x}-2 \cdot 2^{-1 / x}\right)=\left(\frac{3^{-1 / x}}{x^{2}}\right)\left(-(\ln 7) 21^{1 / x}+(\ln 27)-(\ln 4)\left(\frac{2}{3}\right)^{-1 / x}\right)<0 .
$$

Since $\lim _{n \rightarrow \infty}\left(7^{1 / n}+3 \cdot 3^{-1 / n}-2 \cdot 2^{-1 / n}\right)=1+3-2=2$, we are done.

The next theorem shows that if the larger region has three components, then the smaller region has at most five components. When this result is combined with the preceding theorem, we see that when the larger region is disconnected, there can be at most eight distinct components (total).

Theorem 2.8. If the larger region of a double bubble has three components, then the smaller region has at most five components. 
Proof. When $n=3$, we know that the larger region of a double bubble has fewer than three components. When $n \geq 4$, one can show that $G\left(\frac{39}{64}\right)<3^{1 / n}<G\left(\frac{1}{2}\right)$.

Since $G$ is continuous, there exists $v_{0} \in\left(\frac{1}{2}, \frac{39}{64}\right)$ such that $G\left(v_{0}\right)=3^{1 / n}$. Now suppose that the larger region $v$ of a $(v, 1-v)$ double bubble in $\mathbb{R}^{n}$ has three components. Then $G(1-v) \leq$ $G\left(1-v_{0}\right)=2+\left(\frac{v_{0}}{1-v_{0}}\right)^{(n-1) / n}\left[-1+G\left(v_{0}\right)-\left(\frac{1-v_{0}}{v_{0}}\right)^{(n-1) / n}\right]=1+\left(\frac{v_{0}}{1-v_{0}}\right)^{(n-1) / n}\left[-1+3^{1 / n}\right]<$ $1+\left(\frac{39}{25}\right)^{(n-1) / n}\left[-1+3^{1 / n}\right]$.

To finish the proof, show that $\left(1+\left(\frac{39}{25}\right)^{(n-1) / n}\left[-1+3^{1 / n}\right]\right)^{n}<6$ by proving that $6^{1 / n}+$ $\left(\frac{39}{25}\right)\left(\frac{25}{39}\right)^{1 / n}-\left(\frac{39}{25}\right)\left(\frac{75}{39}\right)^{1 / n}>1$.

\section{Preliminaries}

In $\mathbb{R}^{n}$, a standard double bubble consists of three spherical caps which intersect at $120^{\circ}$ angles. It has an axis of symmetry, which will serve as the $x$-axis. If we place the origin at the center of the larger bubble, then in any plane through the $x$-axis, the line from the origin to a point of common intersection forms a well-defined "cap angle" with the $\mathrm{x}$-axis, which we will call $\phi$. Let $R$ be the distance from the origin to a point of common intersection (i.e., the radius of the larger bubble).

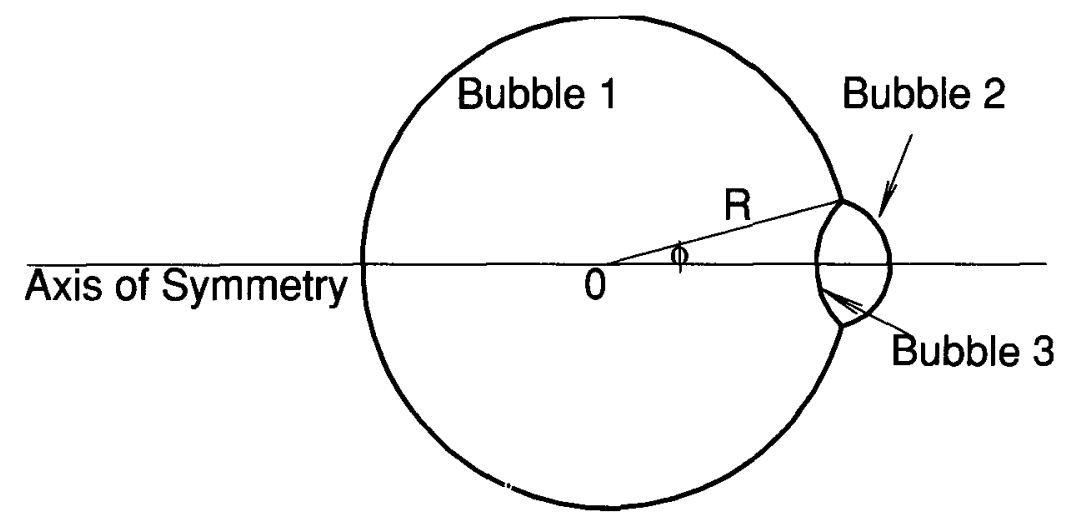

FIGURE 1

Using high school geometry, we can find that:

- The "cap angle" of the second bubble is $\phi_{2}:=\pi / 3+\phi$.

- The radius of the second bubble is $R_{2}:=\frac{\sin (\phi)}{\sin (\pi / 3+\phi)} R$.

- The "cap angle" of the third bubble is $\phi_{3}:=\pi / 3-\phi$.

- The radius of the third bubble is $R_{3}:=\frac{\sin (\phi)}{\sin (\pi / 3-\phi)} R$.

We will also need to calculate a few volumes:

- The volume of the part of the first bubble for which $x \leq R \cos (\phi)$ is

$$
V_{1}:=\int_{-R}^{R \cos \phi} \alpha_{n-1}\left(\sqrt{R^{2}-x^{2}}\right)^{n-1} d x=\alpha_{n-1} R^{n} \int_{0}^{\pi-\phi} \sin ^{n} \theta d \theta
$$


- The volume of the part of the second bubble for which $x \geq R \cos (\phi)$ is

$$
V_{2}:=\int_{-R_{2}}^{R_{2} \cos \phi_{2}} \alpha_{n-1}\left(\sqrt{R_{2}^{2}-x^{2}}\right)^{n-1} d x=\alpha_{n-1}\left(\frac{\sin (\phi)}{\sin (\pi / 3+\phi)}\right)^{n} R^{n} \int_{0}^{\pi / 3+\phi} \sin ^{n} \theta d \theta
$$

- The volume of the part of the third bubble for which $x \leq R \cos (\phi)$ is

$$
V_{3}:=\int_{R_{3} \cos \phi_{3}}^{R_{3}} \alpha_{n-1}\left(\sqrt{R_{3}^{2}-x^{2}}\right)^{n-1} d x=\alpha_{n-1}\left(\frac{\sin (\phi)}{\sin (\pi / 3-\phi)}\right)^{n} R^{n} \int_{0}^{\pi / 3-\phi} \sin ^{n} \theta d \theta
$$

At various points in the proof, it will be convenient to introduce another function,

$$
F(n, \phi):=\frac{\int_{0}^{\phi} \sin ^{n} \theta d \theta}{\sin ^{n} \phi}
$$

We will also need to know a few properties of $F$.

Lemma 3.1. Given $F(n, \phi):=\frac{\int_{0}^{\phi} \sin ^{n} \theta d \theta}{\sin ^{n} \phi}$ and $n \in \mathbb{N}$,

(1) $\frac{\partial}{\partial \phi} F(n, \phi)=1-n \cot (\phi) F(n, \phi) \geq 0 \forall \phi \in(0, \pi)$.

(2) $\frac{\partial^{2}}{\partial \phi^{2}} F(n, \phi) \geq 0 \forall \phi \in(0, \pi)$.

(3) $F(n, \phi) \geq \frac{\sin (\phi)}{n+1} \forall \phi \in(0, \pi)$.

(4) $F(n, \phi) \leq \frac{\tan (\phi)}{n+1} \forall \phi \in(0, \pi / 2)$.

(5) $\lim _{\phi \rightarrow 0} F(n, \phi)=0$.

Proof. To prove statement (3), show that $\int_{0}^{\phi} \sin ^{n} \theta d \theta-\frac{1}{n+1} \sin ^{n+1}(\phi)$ is positive by taking its derivative. Statement (4) can be proven with a similar trick.

Remark. It is interesting to note that after rescaling, $F(n, \phi)$ has a nice geometric interpretation (although this fact will not be used in this article). In particular,

$$
\frac{F(n, \phi)}{\int_{0}^{\pi} \sin ^{n} \theta d \theta}=\frac{\alpha_{n-1} \int_{0}^{\phi} \sin ^{n} \theta d \theta}{\alpha_{n} \sin ^{n} \phi},
$$

which is the ratio of the volume of an $n$-spherical cap to the volume of an entire $n$-sphere whose radius is that of the cap's boundary. This is a nice (and amusing) way to visualize the behavior of $F(n, \phi)$ in low dimensions.

\section{Proof of the curvature conjecture when $w \geq 1$}

To prove the curvature conjecture when $w>1$, we need to show that the curvature of a sphere with volume $V_{1}-V_{3}$, added to the curvature of a sphere with volume $V_{1}+V_{2}$, is less than twice the curvature of a sphere of radius $R$. Thus our goal in this section is to prove that

$$
\sqrt[n]{\frac{\alpha_{n}}{V_{1}+V_{2}}}+\sqrt[n]{\frac{\alpha_{n}}{V_{1}-V_{3}}}<\frac{2}{R}
$$


After substituting, canceling the $R$ 's, and using the identity $\int_{0}^{\pi} \sin ^{n} \theta d \theta=\frac{\alpha_{n}}{\alpha_{n-1}}$, we see that it suffices to prove that

$$
\begin{aligned}
& \sqrt[n]{\frac{\int_{0}^{\pi} \sin ^{n} \theta d \theta}{\int_{0}^{\pi-\phi} \sin ^{n} \theta d \theta+\left(\frac{\sin (\phi)}{\sin (\pi / 3+\phi)}\right)^{n} \int_{0}^{\pi / 3+\phi} \sin ^{n} \theta d \theta}} \\
& +\sqrt[n]{\frac{\int_{0}^{\pi} \sin ^{n} \theta d \theta}{\int_{0}^{\pi-\phi} \sin ^{n} \theta d \theta-\left(\frac{\sin (\phi)}{\sin (\pi / 3-\phi)}\right)^{n} \int_{0}^{\pi / 3-\phi} \sin ^{n} \theta d \theta}}<2 .
\end{aligned}
$$

This still looks pretty complicated, but a lemma will help to simplify things.

Lemma 4.1. If $0 \leq \delta<\epsilon \leq 1$, then $\sqrt[n]{1-\epsilon}+\sqrt[n]{1+\delta}<2 \forall n \in \mathbb{N}$.

Proof. Show that $\sqrt[n]{1-x}+\sqrt[n]{1+x}$ is decreasing on $(0,1)$.

Here, we need to prove a particular instance of $\sqrt[n]{1-\epsilon}+\sqrt[n]{1+\delta}<2$ where $\delta$ and $\epsilon$ are between 0 and 1. Therefore, it is sufficient to prove that $\delta<\epsilon$. This justifies some sleight of hand to remove the root signs! It now suffices to prove that

$$
\frac{\int_{0}^{\pi} \sin ^{n} \theta d \theta}{\int_{0}^{\pi-\phi} \sin ^{n} \theta d \theta+\left(\frac{\sin (\phi)}{\sin (\pi / \beta+\phi)}\right)^{n} \int_{0}^{\pi / \beta+\phi} \sin ^{n} \theta d \theta}+\frac{\int_{0}^{\pi} \sin ^{n} \theta d \theta}{\int_{0}^{\pi-\phi} \sin ^{n} \theta d \theta-\left(\frac{\sin (\phi)}{\sin (\pi \beta-\phi)}\right)^{n} \int_{0}^{\pi \beta-\phi} \sin ^{n} \theta d \theta}<2 .
$$

This is a tighter inequality, but an easier one to prove, since we can now manipulate the terms and turn the inequality around. The following rearrangement will seem clearer if we think of the terms in the preceding equation geometrically, as the ratios of volumes in a double bubble. For convenience, we'll define one more volume, $V_{1 b}:=\alpha_{n}-V_{1}$, and then run through the calculation.

$$
\begin{aligned}
\frac{\alpha_{n}}{V_{1}+V_{2}}+\frac{\alpha_{n}}{V_{1}-V_{3}}<2 & \Longleftrightarrow \frac{V_{1}+V_{2}-\left(V_{2}-V_{1 B}\right)}{V_{1}+V_{2}}+\frac{\left(V_{1}-V_{3}\right)+\left(V_{3}+V_{1 B}\right)}{V_{1}-V_{3}}<2 \\
& \Longleftrightarrow \frac{V_{3}+V_{1 B}}{V_{1}-V_{3}}<\frac{V_{2}-V_{1 B}}{V_{1}+V_{2}} \\
& \Longleftrightarrow \frac{V_{1}-V_{3}}{V_{3}+V_{1 B}}>\frac{V_{1}+V_{2}}{V_{2}-V_{1 B}} \\
& \Longleftrightarrow \frac{\left(V_{1}+V_{1 B}\right)-\left(V_{3}+V_{1 B}\right)}{V_{3}+V_{1 B}}>\frac{\left(V_{1}+V_{1 B}\right)+\left(V_{2}-V_{1 B}\right)}{V_{2}-V_{1 B}} \\
& \Longleftrightarrow \frac{\alpha_{n}}{V_{3}+V_{1 B}}-\frac{\alpha_{n}}{V_{2}-V_{1 B}}>2 .
\end{aligned}
$$

We have just shown that it is sufficient to prove that

$$
\frac{\int_{0}^{\pi} \sin ^{n} \theta d \theta}{\int_{0}^{\phi} \sin ^{n} \theta d \theta+\left(\frac{\sin (\phi)}{\sin (\pi \beta-\phi)}\right)^{n} \int_{0}^{\pi / 3-\phi} \sin ^{n} \theta d \theta}-\frac{\int_{0}^{\pi} \sin ^{n} \theta d \theta}{\left(\frac{\sin (\phi)}{\sin (\pi \beta+\phi)}\right)^{n} \int_{0}^{\pi / 3+\phi} \sin ^{n} \theta d \theta-\int_{0}^{\phi} \sin ^{n} \theta d \theta}>2 .
$$

After re-expressing this in terms of the function $F(n, \phi)$ which was defined in Section 3, we just need to prove the following equivalent assertion.

$$
\frac{1}{\sin ^{n} \phi}\left[\frac{1}{F(n, \phi)+F(n, \pi / 3-\phi)}-\frac{1}{F(n, \pi / 3+\phi)-F(n, \phi)}\right]>\frac{1}{F(n, \pi / 2)} .
$$

This is still pretty complicated, but we can bound the terms in a way that will make the expression reduce into something much simpler.

Lemma 4.2. $F(n, \phi)+F(n, \pi / 3-\phi) \leq F(n, \pi / 3) \forall \phi \in(0, \pi / 3) \forall n \in \mathbb{N}$. 
Proof. $\frac{\partial}{\partial \phi}[F(n, \phi)+F(n, \pi / 3-\phi)]=\frac{\partial F}{\partial \phi}(n, \phi)-\frac{\partial F}{\partial \phi}(n, \pi / 3-\phi)<0 \forall \phi \in(0, \pi / 6)$ because $F$ is concave-up. Thus on $(0, \pi / 6]$, the function has an upper bound at $\lim _{\phi \rightarrow \pi / 3}[F(n, \phi)+$ $F(n, \pi / 3-\phi)]=F(n, \pi / 3)$. By symmetry, this is also true when $\phi \in[\pi / 6, \pi / 3)$.

Lemma 4.3. $\cos (\phi)[F(n, \pi / 3+\phi)-F(n, \phi)] \geq F(n, \pi / 3) \forall \phi \in(0, \pi / 3) \forall n \geq 3$.

Proof. This technical proof is deferred until Section 6, at the end of the article.

When we apply these two lemmas, the problem gets very manageable. It is now sufficient to prove that $\frac{1-\cos (\phi)}{\sin ^{n} \phi}\left[\frac{1}{F(n, \pi / 3)}\right]>\frac{1}{F(n, \pi / 2)}$. One can easily verify that when $n \geq 3, \frac{1-\cos (\phi)}{\sin ^{n} \phi}$ is decreasing on $(0, \pi / 3)$. Therefore, it suffices to prove that $\frac{1-\cos (\pi / 3)}{\sin ^{n} \pi / 3}\left[\frac{1}{F(n, \pi / 3)}\right]>\frac{1}{F(n, \pi / 2)}$. However, it is also easy to show that $\frac{1-\cos (\phi)}{\sin ^{n}(\phi)}\left[\frac{1}{F(n, \phi)}\right]$ is decreasing. Thus $\frac{1-\cos (\pi / 3)}{\sin ^{n}(\pi / 3)}\left[\frac{1}{F(n, \pi / 3)}\right]>$ $\frac{1-\cos (\pi / 2)}{\sin ^{n}(\pi / 2)}\left[\frac{1}{F(n, \pi / 2)}\right]$, which finishes the problem on $(0, \pi / 3)$ for all $n \geq 3$. This completes the proof of the Curvature Conjecture when $w>1$.

To prove the Curvature Conjecture when the two enclosed volumes are equal $(w=1)$, we just need to prove that

$$
\sqrt[n]{\frac{\int_{0}^{\pi} \sin ^{n} \theta d \theta}{2 \int_{0}^{2 \pi / 3} \sin ^{n} \theta d \theta}}+\sqrt[n]{\frac{\int_{0}^{\pi} \sin ^{n} \theta d \theta}{\int_{0}^{2 \pi / 3} \sin ^{n} \theta d \theta}}<2 .
$$

Note that Lemma 4.1 applies, so it suffices to prove $3\left(\frac{\int_{0}^{\pi / 2} \sin ^{n} \theta d \theta}{\int_{0}^{2 \pi / 3} \sin ^{n} \theta d \theta}\right)<2$.

Equivalently, we can simplify and prove that $\int_{0}^{\pi / 3} \sin ^{n} \theta d \theta<\int_{\pi / 3}^{\pi / 2} \sin ^{n} \theta d \theta$. Since $\int_{0}^{\pi / 3} \sin ^{n} \theta d \theta<\sin ^{n-1}\left(\frac{\pi}{3}\right) \int_{0}^{\pi / 3} \sin \theta d \theta=\sin ^{n-1}\left(\frac{\pi}{3}\right) \int_{\pi / 3}^{\pi / 2} \sin \theta d \theta<\int_{\pi / 3}^{\pi / 2} \sin ^{n} \theta d \theta \forall n \geq 2$, the proof is complete.

\section{Proof of the curvature conjecture when $w<1$}

To prove the curvature conjecture in the case when $w<1$, we need to show that the curvature of a sphere with volume $V_{2}+V_{3}$, added to the curvature of a sphere with volume $V_{1}+V_{2}$, is less than twice the curvature of a sphere of radius $R_{2}$ (where $R_{2}=\frac{\sin (\phi)}{\sin (\pi / 3+\phi)} R$ ). Thus our goal is to prove that

$$
\sqrt[n]{\frac{\alpha_{n}}{V_{1}+V_{2}}}+\sqrt[n]{\frac{\alpha_{n}}{V_{2}+V_{3}}}<\frac{2}{R_{2}} .
$$

If we substitute and cancel the $R_{2}$ 's, and denote

$$
f_{1}(n, \phi):=\frac{\int_{0}^{\pi} \sin ^{n} \theta d \theta}{\left(\frac{\sin (\pi / 3+\phi)}{\sin (\phi)}\right)^{n} \int_{0}^{\pi-\phi} \sin ^{n} \theta d \theta+\int_{0}^{\pi / 3+\phi} \sin ^{n} \theta d \theta}
$$

and

$$
f_{2}(n, \phi):=\frac{\int_{0}^{\pi} \sin ^{n} \theta d \theta}{\int_{0}^{\pi / 3+\phi} \sin ^{n} \theta d \theta+\left(\frac{\sin (\pi / 3+\phi)}{\sin (\pi / 3-\phi)}\right)^{n} \int_{0}^{\pi / 3-\phi} \sin ^{n} \theta d \theta},
$$

this is equivalent to proving that $\sqrt[n]{f_{1}}+\sqrt[n]{f_{2}}<2$. 
Lemma 5.1. (1) $\sqrt[n]{f_{1}(n, \phi)}<\frac{1}{2} \forall \phi \in(0, \pi / 6] \quad \forall n \in \mathbb{N}$.

$$
\text { (2) } \sqrt[n]{f_{1}(n, \phi)}<\frac{3}{4} \forall \phi \in[\pi / 6, \pi / 4] \forall n \in \mathbb{N} \text {. }
$$

Proof. Since $\frac{\partial}{\partial \phi} \sqrt[n]{f_{1}(n, \phi)}>0$, it suffices to check the right endpoint of each interval.

(1) We can prove that $f_{1}\left(n, \frac{\pi}{6}\right)<\left(\frac{1}{2}\right)^{n}$ by noting that

$$
2^{n} \int_{0}^{5 \pi / 6} \sin ^{n} \theta d \theta+\int_{0}^{\pi / 2} \sin ^{n} \theta d \theta=2^{n} \int_{0}^{\pi} \sin ^{n} \theta d \theta-F(n, \pi / 6)+F(n, \pi / 2),
$$

which is greater than $2^{n} \int_{0}^{\pi} \sin ^{n} \theta d \theta$ because $F$ is increasing. Therefore,

$$
f_{1}\left(n, \frac{\pi}{6}\right)=\frac{\int_{0}^{\pi} \sin ^{n} \theta d \theta}{2^{n} \int_{0}^{5 \pi / 6} \sin ^{n} \theta d \theta+\int_{0}^{\pi / 2} \sin ^{n} \theta d \theta}<\frac{\int_{0}^{\pi} \sin ^{n} \theta d \theta}{2^{n} \int_{0}^{\pi} \sin ^{n} \theta d \theta}=\left(\frac{1}{2}\right)^{n} .
$$

To prove (2), note that $f_{1}\left(n, \frac{\pi}{4}\right)$ is equal to

$$
\frac{\int_{0}^{\pi} \sin ^{n} \theta d \theta}{\left(\frac{\sqrt{3}+1}{2}\right)^{n} \int_{0}^{\pi} \sin ^{n} \theta d \theta+\sin ^{n}\left(\frac{7 \pi}{12}\right)\left[-F\left(n, \frac{\pi}{4}\right)+F\left(n, \frac{7 \pi}{12}\right)\right]}<\frac{\int_{0}^{\pi} \sin ^{n} \theta d \theta}{\left(\frac{\sqrt{3}+1}{2}\right) \int_{0}^{n} \sin ^{n} \theta d \theta}<\left(\frac{3}{4}\right)^{n} .
$$

Lemma 5.2. (1) $\sqrt[n]{f_{2}(n, \phi)}<\frac{3}{2} \forall \phi \in(0, \pi / 6] \quad \forall n \geq 3$.

$$
\text { (2) } \sqrt[n]{f_{2}(n, \phi)}<\frac{5}{4} \forall \phi \in[\pi / 6, \pi / 4] \forall n \geq 3 \text {. }
$$

Proof. By taking a derivative, one can verify that the left-hand side is a decreasing function. Therefore, it suffices to check the left endpoint of each subinterval.

$$
\text { To prove (1), it is sufficient to prove that } f_{2}(n, 0)=\frac{\int_{0}^{\pi} \sin ^{n} \theta d \theta}{2 \int_{0}^{\pi / 3} \sin ^{n} \theta d \theta}<\left(\frac{3}{2}\right)^{n} \text {. }
$$

When $n \in\{3,4,5\}$, one can just verify this by direct calculation. Here is the proof for $n \geq 6$. By property (3) from Lemma 3.1, it suffices to prove that

$$
\frac{\int_{0}^{\pi} \sin ^{n} \theta d \theta}{\frac{2}{n+1} \sin ^{n+1}(\pi / 3)}<\left(\frac{3}{2}\right)^{n}
$$

Equivalently, we need to prove that $\int_{0}^{\pi} \sin ^{n} \theta d \theta<\left(\frac{\sqrt{3}}{n+1}\right)\left(\frac{3 \sqrt{3}}{4}\right)^{n}$. For $n \geq 6$, this is easy to verify, since the left-hand side is less than one and the right-hand side is greater than one.

To prove (2), it suffices to show that

$$
\frac{\int_{0}^{\pi} \sin ^{n} \theta d \theta}{\int_{0}^{\pi / 2} \sin ^{n} \theta d \theta+\left(\frac{1}{\sin (\pi / 6)}\right)^{n} \int_{0}^{\pi / 6} \sin ^{n} \theta d \theta}<\left(\frac{5}{4}\right)^{n} .
$$

When $n=3$, one can just verify this by direct calculation. More generally, the left-hand side is clearly less than 2 , which is less than $\left(\frac{5}{4}\right)^{n}$ for all $n \geq 4$.

When Lemmas 5.1 and 5.2 are combined, we have a proof of the Curvature Conjecture for $\phi \in(0, \pi / 4]$. When $\phi \in(\pi / 4, \pi / 3)$, the conditions for Lemma 4.1 hold, so we can remove the 
root signs. Thus it is now sufficient to prove that

$$
\frac{\int_{0}^{\pi} \sin ^{n} \theta d \theta}{\left(\frac{\sin (\pi / 3+\phi)}{\sin (\phi)}\right)^{n} \int_{0}^{\pi-\phi} \sin ^{n} \theta d \theta+\int_{0}^{\pi / 3+\phi} \sin ^{n} \theta d \theta}+\frac{\int_{0}^{\pi} \sin ^{n} \theta d \theta}{\int_{0}^{\pi / 3+\phi} \sin ^{n} \theta d \theta+\left(\frac{\sin (\pi \beta+\phi)}{\sin (\pi / \beta-\phi)}\right)^{n} \int_{0}^{\pi / 3-\phi} \sin ^{n} \theta d \theta}<2 .
$$

The next two lemmas will prove this for all $\phi \in(\pi / 4, \pi / 3)$ and $n \geq 3$.

Lemma 5.3. $f_{2}(n, \phi)<\frac{14}{10} \forall \phi \in(\pi / 4, \pi / 3) \forall n \geq 3$.

Proof. Since the left-hand side is decreasing, it suffices to prove that

$$
f_{2}\left(n, \frac{\pi}{4}\right)=\frac{\int_{0}^{\pi} \sin ^{n} \theta d \theta}{\int_{0}^{7 \pi / 12} \sin ^{n} \theta d \theta+\left(\frac{\sin (7 \pi / 12)}{\sin (\pi / 12)}\right)^{n} \int_{0}^{\pi / 12} \sin ^{n} \theta d \theta}<\frac{14}{10}
$$

When $n=3$ or $n=4$, one can verify this by direct calculation. To finish the proof, we can use trigonometric reduction to show that $f_{2}(n+2, \pi / 4) \leq f_{2}(n, \pi / 4)$. Since $f_{2}(n+2, \pi / 4)$ is equal to

$$
\frac{\int_{0}^{\pi} \sin ^{n} \theta d \theta}{-\frac{\sin ^{n+1}(7 \pi / 12) \cos \frac{7 \pi}{12}}{n+1}-\frac{\sin ^{n+2}(7 \pi / 12) \cot \frac{\pi}{12}}{n+1}+\int_{0}^{\frac{7 \pi}{12}} \sin ^{n} \theta d \theta+\left(\frac{\sin (7 \pi / 12)}{\sin (\pi / 12)}\right)^{n+2} \int_{0}^{\frac{\pi}{12}} \sin ^{n} \theta d \theta},
$$

it suffices to prove that

$$
-\frac{\sin ^{n+1}(7 \pi / 12) \cos \frac{7 \pi}{12}}{n+1}-\frac{\sin ^{n+2}(7 \pi / 12) \cot \frac{\pi}{12}}{n+1}+\left(\frac{\sin (7 \pi / 12)}{\sin (\pi / 12)}\right)^{n+2} \int_{0}^{\frac{\pi}{12}} \sin ^{n} \theta d \theta \geq\left(\frac{\sin (7 \pi / 12)}{\sin (\pi / 12)}\right)^{n} \int_{0}^{\frac{\pi}{12}} \sin ^{n} \theta d \theta .
$$

Equivalently, we need to prove that

$$
\left(\frac{\sin ^{2}(7 \pi / 12)-\sin ^{2}(\pi / 12)}{\sin ^{2}(\pi / 12)}\right) \frac{\int_{0}^{\frac{\pi}{12}} \sin ^{n} \theta d \theta}{\sin ^{n}(\pi / 12)} \geq\left(\frac{\sin (7 \pi / 12)}{n+1}\right) \frac{\sin (\pi / 3)}{\sin (\pi / 12)} .
$$

Simplifying again, we need to prove that $\left(\frac{\sin (\pi / 3)}{\sin (\pi / 12)}\right) F(n, \pi / 12) \geq \frac{\sin (7 \pi / 12) \sin (\pi / 3)}{n+1}$. However, since $F(n, \pi / 12) \geq \frac{\sin (\pi / 12)}{n+1}$ by part (3) of Lemma 3.1, the proof is done.

Lemma 5.4. $f_{1}(n, \phi)<\frac{6}{10} \forall \phi \in(\pi / 4, \pi / 3) \forall n \geq 3$.

Proof. Since the left-hand side is increasing, it is sufficient to check when $\phi=\pi / 3$. When $n=3$ or $n=4$, one can verify this by direct calculation. For higher $n$, one can use trigonometric reduction to show that the function decreases as $n$ increases.

This completes the proof of the curvature conjecture when $w<1$.

\section{Proof of Lemma 4.3}

Before getting to the actual proof, we will need two technical lemmas, whose value will be apparent later. It is probably best to just skip them for now, and refer back to them as necessary.

Lemma 6.1. $[\tan (\phi)+n \cot (\phi)] F(n, \phi) \geq \frac{n}{n+1} \forall \phi \in(0, \pi / 2) \forall n \in \mathbb{N}$. 
Proof. Let $h(\phi):=\int_{0}^{\phi} \sin ^{n} \theta d \theta-\left(\frac{n}{n+1}\right) \frac{\sin ^{n+1} \phi \cos \phi}{1+(n-1) \cos ^{2} \phi}$. Then $h(0)=0$, and $\frac{d h}{d \phi}$ is equal to $\frac{\sin ^{n+2} \phi}{\left[1+(n-1) \cos ^{2} \phi\right]^{2}}\left\{\left[1+(n-1) \cos ^{2} \phi\right]\left[1-\cos ^{2} \phi+\frac{n}{n+1} \sin ^{2} \phi\right]-\frac{2 n(n-1)}{n+1} \sin ^{2} \phi \cos ^{2} \phi\right\}$, which simplifies to $\frac{\sin ^{n+2}(\phi)}{\left[1+(n-1) \cos ^{2} \phi\right]^{2}}\left(\frac{2 n+1}{n+1}+\frac{n-1}{n+1} \cos ^{2} \phi\right) \geq 0$. Thus $h(\phi) \geq 0 \forall \phi \in\left(0, \frac{\pi}{2}\right)$. The result follows when we multiply $h(\phi)$ by $\frac{\tan (\phi)+n \cot (\phi)}{\sin ^{n}(\phi)}$.

Lemma 6.2. When $n \geq 3$ and $\phi \in[0, \pi / 3]$, $-\frac{2 n+1}{2}-\left(n^{2}-2 n-2\right) \cos \phi \cos (\pi / 3+\phi)+2 n(n-1) \cos ^{2} \phi+2(n-1) \cos ^{2} \phi \cos ^{2}(\pi / 3+\phi) \geq 0$.

Proof. Since $\cos (x)=\cos (\pi / 3+x)+\cos (\pi / 3-x)$, we can equivalently prove that

$$
\begin{aligned}
& -\frac{2 n+1}{2}+\left(n^{2}-2 n+4\right) \cos (\phi) \cos (\pi / 3+\phi)+2 n(n-1) \cos (\phi) \cos (\pi / 3-\phi) \\
& +2(n-1) \cos (\phi) \cos (\pi / 3+\phi)[1+\cos (\phi) \cos (\pi / 3+\phi)] \geq 0 .
\end{aligned}
$$

By taking derivatives, we can verify that

(1) $\cos (\phi) \cos (\pi / 3+\phi)$ is decreasing on $(0, \pi / 3)$.

(2) $\cos (\phi) \cos (\pi / 3-\phi)$ increases on $(0, \pi / 6)$ and decreases on $(\pi / 6, \pi / 3)$.

(3) $\cos (\phi) \cos (\pi / 3+\phi)[1+\cos (\phi) \cos (\pi / 3+\phi)]$ is decreasing on $(0, \pi / 3)$.

Thus when $\phi \in[0, \pi / 6]$, it suffices to show that $-\frac{2 n+1}{2}+0+2 n(n-1) \cos (0) \cos \left(\frac{\pi}{3}\right) \geq 0$. But this is equal to $n^{2}-2 n-\frac{1}{2}$, which is positive for all $n \geq 3$. Similarly, since the function is decreasing on the entire interval $[\pi / 6, \pi / 3]$, we can finish the proof by verifying the proposition at the right endpoint. Fortunately, the value at $\pi / 3$ is $-\frac{2 n+1}{2}+\left(n^{2}-2 n+4\right)\left(\frac{1}{2}\right)\left(-\frac{1}{2}\right)+2 n(n-1)\left(\frac{1}{2}\right)+$ $2(n-1)\left(\frac{1}{2}\right)\left(-\frac{1}{2}\right)\left[1+\left(\frac{1}{2}\right)\left(-\frac{1}{2}\right)\right]$, which is equal to $\left(\frac{3}{8}\right)\left[2 n^{2}-5 n-3\right]$, which is nonnegative for all $n \geq 3$.

\section{Proof of Lemma 4.3}

Now we can prove Lemma 4.3 , which states that

$$
\cos (\phi)[F(n, \pi / 3+\phi)-F(n, \phi)] \geq F(n, \pi / 3) \forall \phi \in(0, \pi / 3) \forall n \geq 3 .
$$

The strategy is to show that $\cos (\phi)[F(n, \pi / 3+\phi)-F(n, \phi)]$ is increasing on $(0, \pi / 3)$. Since the function is continuous, and $\lim _{\phi \rightarrow 0}[F(n, \pi / 3+\phi)-F(n, \phi)]=F(n, \pi / 3)$, this will prove that $F(n, \pi / 3)$ is a lower bound. $\frac{d}{d \phi}(\cos (\phi)[F(n, \pi / 3+\phi)-F(n, \phi)])$ is equal to

$$
-\sin \phi\left[F\left(n, \frac{\pi}{3}+\phi\right)-F(n, \phi)\right]+\cos \phi\left[-n \cot \left(\frac{\pi}{3}+\phi\right) F\left(n, \frac{\pi}{3}+\phi\right)+n \cot \phi F(n, \phi)\right] .
$$

Thus we can show that $\cos (\phi)[F(n, \pi / 3+\phi)-F(n, \phi)]$ is increasing by proving that

$$
[\tan (\phi)+n \cot (\pi / 3+\phi)] F(n, \pi / 3+\phi) \leq[\tan (\phi)+n \cot (\phi)] F(n, \phi) .
$$

By Lemma 6.1, it suffices to show $[\tan (\phi)+n \cot (\pi / 3+\phi)] F(n, \pi / 3+\phi) \leq \frac{n}{n+1}$.

When $n=3, \tan (\phi)+n \cot (\pi / 3+\phi)$ is clearly positive on $(0, \pi / 3)$. When $n \geq 4$, the decreasing function $\tan (\phi)+n \cot (\pi / 3+\phi)$ becomes negative for $\phi$ near $\pi / 3$. Since our inequality is automatically satisfied when $\tan (\phi)+n \cot (\pi / 3+\phi) \leq 0$, negative values are not 
a problem! On the interval where $\tan (\phi)+n \cot (\pi / 3+\phi)>0$, we can verify the inequality by rearranging it as follows:

$$
\begin{aligned}
& {[\tan (\phi)+n \cot (\pi / 3+\phi)] F(n, \pi / 3+\phi) \leq \frac{n}{n+1}} \\
& \quad \Longleftrightarrow \frac{n+1}{n} \int_{0}^{\pi / 3+\phi} \sin ^{n} \theta d \theta \leq \frac{\sin ^{n}(\pi / 3+\phi)}{\tan (\phi)+n \cot (\pi / 3+\phi)} \\
& \quad \Longleftrightarrow f(\phi):=\frac{\sin ^{n+1}(\pi / 3+\phi) \cos (\phi)}{\frac{1}{2}+(n-1) \cos (\phi) \cos (\pi / 3+\phi)}-\left(\frac{n+1}{n}\right) \int_{0}^{\pi / 3+\phi} \sin ^{n} \theta d \theta \geq 0 .
\end{aligned}
$$

By property (4) of Lemma 3.1, $f(0)=\left(\frac{1}{n}\right) \sin ^{n}\left(\frac{\pi}{3}\right)\left[\tan \left(\frac{\pi}{3}\right)-(n+1) F\left(n, \frac{\pi}{3}\right)\right] \geq 0$. Therefore, it is sufficient to show that $f(\phi)$ is increasing on $(0, \pi / 3)$. So we take its derivative! After some simplification, one can show that $\frac{d f}{d \phi}$ is equal to

$$
\frac{\left[-\frac{2 n+1}{2}-\left(n^{2}-2 n-2\right) \cos \phi \cos \left(\frac{\pi}{3}+\phi\right)+2 n(n-1) \cos ^{2} \phi+2(n-1) \cos ^{2} \phi \cos ^{2}\left(\frac{\pi}{3}+\phi\right)\right] \sin ^{n}\left(\frac{\pi}{3}+\phi\right)}{2 n\left[\frac{1}{2}+(n-1) \cos \phi \cos \left(\frac{\pi}{3}+\phi\right)\right]^{2}} .
$$

But we know from Lemma 6.2 that this is nonnegative, so the proof is complete.

\section{Acknowledgments}

The author first encountered this problem at the Clay Research Institute on the Global Theory of Minimal Surfaces which was held at MSRI in 2001, and she thanks them for their hospitality. The author would particularly like to thank Frank Morgan for introducing her to this problem, and for his help and encouragement.

\section{References}

[1] Almgren, Jr., F. J. Existence and regularity almost everywhere of solutions to elliptic variational problems with constraints, Memoirs AMS 165(4), (1976).

[2] Corneli, J., Hoffman, N., Holt, P., Lee, G., Leger, N., Moseley, S., and Schoenfeld, E. Double bubbles in $S^{3}$ and $H^{3}, J$. Geom. Anal., to appear.

[3] Foisy, J., Alfaro, M., Brock, J., Hodges, N., and Zimba, J. The standard double bubble in $\mathbb{R}^{2}$ uniquely minimizes perimeter Pacific J. Math. 159, 47-59, (1993).

[4] Futer, D. Progress on the Curvature Problem, http: //math.msu. edu/ dfuter/curvature/.

[5] Heilmann, C., Lai, Y., Reichardt, B., and Spielman, A. Component bounds for area-minimizing double bubbles, NSF "SMALL" undergraduate research report, Williams College, (1999).

[6] Hutchings, M. The structure of area-minimizing double bubbles, J. Geom. Anal. 7(2), 285-304, (1997).

[7] Hutchings, M., Morgan, F., Ritoré, M., and Ros, A. Proof of the double bubble conjecture, Ann. Math. 155(2), 459-489, (2002).

[8] Morgan, F. Geometric Measure Theory: A Beginner's Guide, Academic Press, 3rd ed., 2000.

[9] Plateau, J. Statique Expérimentale et Théorique des Liquides Soumis aux Seules Forces Moléculaires, Paris, Gauthier-Villars, 1883.

[10] Reichardt, B., Heilmann, C., Lai, Y., and Spielman, A. Proof of the double bubble conjecture in $\mathbb{R}^{4}$ and certain higher dimensional cases, Pacific J. Math. 208, 347-366, (2003).

Received October 10, 2004

Revision received August 24, 2005

Max-Planck-Institute for Gravitational Physics, D-14476 Golm, Germany

e-mail: Marilyn.Daily@aei.mpg.de 